\title{
Kernos
}

Revue internationale et pluridisciplinaire de religion grecque antique

$18 \mid 2005$

Varia

\section{Religion publique et croyances personnelles : Platon contre Socrate?}

\author{
Aikaterini Lefka
}

\section{(2) OpenEdition \\ Journals}

Édition électronique

URL : http://journals.openedition.org/kernos/901

DOI : 10.4000/kernos.901

ISSN : 2034-7871

Éditeur

Centre international d'étude de la religion grecque antique

Édition imprimée

Date de publication : 1 janvier 2005

Pagination : 85-95

ISSN : 0776-3824

Référence électronique

Aikaterini Lefka, « Religion publique et croyances personnelles : Platon contre Socrate? », Kernos [En ligne], 18 | 2005, mis en ligne le 16 juin 2011, consulté le 01 mai 2019. URL : http:// journals.openedition.org/kernos/901; DOI : 10.4000/kernos.901 


\title{
Religion publique et croyances personnelles : Platon contre Socrate ?*
}

\begin{abstract}
Résumé : Cet article examine l'attitude de Socrate (condamné, entre autres, pour sa divergence d'avec les conceptions religieuses de la cité) tel qu'il se présente dans les dialogues platoniciens, face à la religion officielle d'Athènes. Ensuite, nous présentons les positions adoptées par Platon dans ses cités idéales concernant les citoyens qui s'écartent des attitudes religieuses établies par la loi. Enfin, nous mettons en évidence les similitudes et les différences entre Socrate et Platon sur les rapports entre la religion publique et les croyances personnelles. Contrairement aux avis courants, nous défendons l'idée que Platon est resté fidèle du début jusqu'à la fin de son oeuvre aux principes socratiques concernant le divin. En fait, dans ses cités idéales, Platon «renverse » les rôles : les croyances de Socrate, définies dialectiquement par le logos, deviennent le fondement de la législation et sont les seules acceptées officiellement.
\end{abstract}

Abstract: Public Religion and personal beliefs. Plato against Socrates? The present paper examines first Socrates' attitude (condemned, among others, for his non-adherence to the city's religious beliefs) towards the official religion of Athens, as it appears in the Platonic dialogues. We then analyse Plato's position concerning the citizens who diverged from the religious representations established by law in the ideal cities. Finally, we formulate what we consider to be the similarities and differences between ('the Platonic') Socrates and Plato himself concerning the relations between public religion and personal beliefs. Against the current interpretations, we defend the idea that Plato conserved from the beginning till the end of his work his devotion to Socratic principles concerning divinity. In fact, in the ideal cities Plato "reverses" the roles: the intimate convictions of Socrates regarding the gods, defined dialectically by the logos, become the foundation of legislation and are the only ones officially accepted.

\section{Introduction}

Parmi les philosophes grecs, il en est un qui a payé de sa vie l'accusation de ne pas croire aux dieux de la cité, mais d'y introduire des nouveaux daimonia, couplée avec celle de corrompre la jeunesse ${ }^{1}$. Socrate réfuta les deux calomnies, mais ne manqua pas pour autant d'adopter ouvertement une attitude provocante devant ses juges, en défendant une manière personnelle

* Nous aimerions dédier le présent article à Madame Toula Vassilakou-Fassea, dont le soutien à toute épreuve, professionnel et amical, a largement et concrètement facilité notre avancement dans la recherche philosophique pendant les six dernières années. Qu'elle en soit vivement remerciée.

1 Platon, Apologie de Socrate, $24 \mathrm{~b}-\mathrm{c}$. 
de comprendre et de pratiquer la piété qui pouvait ne pas coïncider avec leur avis : "J'obéirai au dieu plutôt qu'à vous »².

L'un de ses élèves, Platon, fut si profondément marqué par cette mise à mort, qu'il consacra trois de ses ouvres à la défense du condamné (Apologie de Socrate), à son refus de s'évader (Criton) et aux dernières heures de son maittre (Phédon). Plus encore, Socrate sera dans la quasi-totalité des dialogues platoniciens l'incontournable protagoniste et l'archétype du philosophe.

Un autre élève, Xénophon, a également voulu vénérer la mémoire de Socrate dans sa propre Apologie et dans ses Mémorables. Comme nous nous concentrerons plus particulièrement dans la présente étude sur d'éventuelles contradictions dans l'ensemble de la pensée de Platon, nous ne prendrons pas en considération ces textes intéressants pour le personnage historique de Socrate lui-même.

En effet, dans sa dernière œuvre, les Lois (dont, exceptionnellement, Socrate est absent), Platon traite avec la plus grande sévérité ceux qui ne se conforment pas aux croyances officielles sur les dieux, dans le cadre de la cité projetée des Magnètes ${ }^{3}$. Sa législation est particulièrement attentive à définir en détail les possibles transgressions et les peines qu'elles méritent. Celles-ci incluent non seulement l'emprisonnement et la «ré-éducation», mais aussi la mise à mort et, pire encore pour les mœurs de l'époque, l'abandon de la dépouille hors des frontières de la cité, sans sépulture, avec interdiction pour tous d'offrir les honneurs dus aux défunts.

Que s'est-il passé, se demandent les chercheurs, entre les dialogues de la jeunesse ou de l'âge mûr et les œuvres de vieillesse, pour que l'esprit d'Antigone qui caractérisait Socrate soit remplacé par celui de Créon dans les préférences du philosophe? Et la plupart concluent que, s'étant détaché de l'influence de son maître, en l'oubliant peut-être avec le temps, Platon exprime finalement son opposition radicale à l'attitude «personnalisée » de Socrate envers la religion publique ${ }^{4}$.

2 Ibid., 29d 3-4. Voir A. MOTTE, "'J'obéirai au dieu plutôt qu'à vous' (Platon, Apologie, 29d) », in M.É. Henneau, C. Havelange, Ph. Denis et J.-P. Delville (éds), Temps, Culture, Religions. Autour de J.-P. Massaut, Louvain-la-Neuve, 2004 (Bibliothèque de la Revue d'Histoire Ecclésiastique, 85), p. 35-47, un article que l'auteur a eu l'amabilité de nous communiquer avant sa publication : l'attitude de Socrate ne saurait être considérée comme répréhensible en soi (quoi de plus pieux que de se soumettre aux commandements divins ?), mais le contexte dans lequel elle s'affiche, qui met en fait les Athéniens en opposition avec la divinité, et la manière provocatrice du philosophe de proférer ces mots, suscitent des réactions fortes chez les juges.

3 Platon, Lois X, 907b-910e.

4 L'hypothèse selon laquelle la pensée de Platon a évolué suite à un «éloignement » de l'enseignement socratique, sous divers aspects, a été notamment défendue par l'éminent G. Vlastos dans plusieurs de ses articles. Quant à l'attitude finale de Platon, considérée comme radicalement opposée à celle de Socrate en ce qui concerne la religion et la liberté des croyances dans la cité, voir, parmi d'autres, L. STRAuss, The Argument and the Action in Plato's Laws, Chicago, 1975; A.W. Nightingale, «Writing/reading a sacred text: A literary interpretation of Plato's Laws », CPh 88 (1993), p. 269-300. 
Nous nous proposons ici de prendre en considération quelques données supplémentaires propres à clarifier davantage cette question délicate et à montrer que certaines déductions courantes méritent une remise en question. À cette fin, nous examinerons d'abord globalement l'attitude de Socrate face à la religion traditionnelle, telle qu'elle se présente dans les dialogues platoniciens. En deuxième lieu, nous nous pencherons sur la position de Platon à l'égard de ceux qui, dans ses cités idéales, s'écartent des croyances officielles, en accordant de l'attention aussi à ce que Socrate dit dans la République. Nous prendrons enfin parti au sujet d'éventuelles contradictions entre Socrate, tel que Platon le représente, et Platon lui-même. Aussi espérons-nous apporter un éclairage supplémentaire concernant la manière dont les Grecs concevaient les aspects du "public» et du "privé » en matière de croyances religieuses.

\section{L'attitude de Socrate envers la religion officielle d'Athènes}

Socrate rejetait-il réellement la religion officielle de l'Athènes de son époque? Dans l'Apologie, il détourne habilement l'accusation de Mélétos, pour refuser l'affirmation selon laquelle il serait un athée complet, lequel pourrait être injustement confondu avec les sophistes ou les penseurs dits «matérialistes ». Pour ce faire, il affirme sa croyance, d'une part, en la divinité des corps célestes (remise en question par Anaxagore, qui fut déjà chassé d'Athènes pour son impiété), et, d'autre part, en l'existence de daimones, compris dans le sens de divinités mineures, ou d'«enfants de dieux».

Nous connaissons l'ambiguité de ce terme pour les Grecs, qui pouvaient y avoir recours tant comme un synonyme du mot theos - soit pour désigner un dieu particulier, soit pour se référer à la divinité en général -, que pour nommer effectivement les divinités mineures.

Socrate se réclame ici des croyances courantes, afin d'éviter de faire passer pour une innovation son «signal divin» (daimonion sémeion). Le philosophe n'hésite pas, d'ailleurs, dans son apologie ${ }^{6}$, à faire ouvertement référence à ce «message divin» qu'il recevait personnellement quand il venait de (ou quand il allait) dire ou faire quelque chose qu'il ne devait pas, et qui était du genre à provoquer des conséquences néfastes. Nous n'avons pas ici le temps de nous arrêter davantage sur la nature et la signification du daimonion socratique, un sujet toujours en discussion? ${ }^{7}$. Pour notre enquête actuelle, qu'il suffise d'en

5 Platon, Apologie, 26b-28a. Pour cette manœeuvre dialectique de Socrate, voir aussi L. Brisson, Platon, Apologie de Socrate, Criton, Paris, GF-Flammarion, 1997, p. 38-39.

6 Platon, Apologie, 40a-c. Nous trouvons d'autres mentions du daimonion dans : Alcibiade I, $103 \mathrm{a}$ 4-b 2; Euthyphron, 3b 5-7; Euthydème, 272e 3-4; République VI, 496c 3-4; Phèdre, 242b 8-9; Théétète, 151a 4. Dans 1' Hippias majeur, 304c 1-4, il est question d'une daimonia tuché qui " possède » le philosophe et l'oblige à errer dans une aporia constante.

Cf., par exemple, J. Burnet, Plato's Euthyphro, Apology of Socrates and Crito, Oxford, 1924, p. 16-17; H. GUNDERT, "Platon und das Daimonion des Sokrates », Gymnasium 61 (1954), p. 513- 
retenir les points suivants : Socrate n'a jamais prétendu que ce signe provenait d'une nouvelle divinité, ni ne l'a identifié à une divinité quelconque (même si le terme daimonion, en tant que substantif, peut aussi signifier «la divinité »). Il ne lui a jamais non plus accordé un culte particulier. Il paraît plutôt ranger cette présence occasionnelle parmi les manières diverses dont les dieux communiquent avec les hommes, comme le sont aussi les oracles ou les rêves. Platon souligne que Socrate essaye d'interpréter correctement ce signe, ce qui implique de sa part une réception «active» et personnalisée, où sa propre intelligence joue un rôle décisif.

Il est vrai que Socrate revendique un rapport particulier et privilégié avec le monde divin, notamment avec un dieu très vénéré par tous les Grecs, Apollon. Dans l'Apologie , mais aussi dans le Phédon', le philosophe présente l'ensemble de son activité philosophique et de sa vie comme une requête d'Apollon en personne, exprimée, d'après l'interprétation que Socrate en avait fait, par un oracle delphique accordé à Chéréphon : "Socrate est le plus sage des hommes ».

En soulignant sa soumission pieuse à un tel dieu, Socrate visait probablement à écarter complètement l'accusation de ne pas vénérer les mêmes dieux que ses concitoyens. En d'autres occasions, il paraît se mettre aussi sous les auspices d'autres grandes divinités, tels Zeus ${ }^{10}$, Héra ${ }^{11}$ ou Artémis ${ }^{12}$.

D'autre part, le Socrate de Platon se lie volontiers à des divinités plus marginales, mais acceptées, pourtant, en tant que dieux par la communauté athénienne. Quand il se présente en prière dans les dialogues, donc quand il effectue l'acte du culte le plus personnel qui soit, le philosophe s'adresse de préférence à des divinités mineures (des daimones), comme Éros et Pan dans

531; W.K.C. GuTHRIE, Socrates, Cambridge, 1971, p. 82-85; A. GomEZ-LoBO, Les Fondements de l'éthique socratique (trad. par N. Ooms), Paris, Presses Universitaires du Septentrion, 1996, p. 7580; Th. C. BrickHouse \& N. D. SMITH, Socrates on Trial, Oxford, 1989, p. 35 et n. 126, p. 242-245; E. De STRYCKer \& S.R. SLIngS, Plato's Apology of Socrates. A Literary and Philosophical Study with a Running Commentary, Leiden, et al., Brill, 1994, p. 153-154; Chr. SCHEFER, Platon und Apollon: vom Logos zurück zum Mythos, Sankt Augustin, Akademia Verlag, 1996 (International Plato Studies, 7), p. 101-108 et le récent et très éclairant article de L.-A. DORION, «Socrate, le daimonion et la divination ", in J. LAURENT (éd.), Les dieux de Platon. Actes du colloque organisé à l'Université de Caen Basse-Normandie les 24, 25 et 26 janvier 2002, Presses Universitaires de Caen, 2003, p. 168-192.

${ }^{8}$ Platon, Apologie, 23a-c; 29c-d; 29d-30a; 30e-31c.

9 Id., Phédon, 84e-85b.

10 Le dieu des philosophes est Zeus dans le Phèdre

11 Socrate avait l'habitude de jurer souvent «par Héra », en lui accordant ainsi le rôle d'une divinité protectrice. Nous nous permettons de renvoyer, pour davantage d'informations, à notre article « 'Par Zeus !'. Les jurons de Platon », Revue de Philosophie Ancienne 21 (2003), p. 54-84.

12 L'art maïeutique est accordé à Socrate par Artémis, qui préside aux accouchements, dans le Théétète, 149b-c. Pour le lien du philosophe avec ces dieux et avec d'autres divinités encore, voir A. LEFKA, "La présence des divinités traditionnelles dans l'œuvre de Platon », in LAURENT (éd.), o.c. (n. 7), p. 97-117 (surtout p. 105-113). 
le $P h e ̀ d r e^{13}$ et au soleil levant selon le témoignage d'Alcibiade dans le Banquet $^{14}$. Quant à son respect des traditions rituelles, ajoutons qu'avant de boire la ciguë, Socrate se soucie de savoir s'il est permis d'offrir les libations habituelles avec le contenu de sa coupe ${ }^{15}$. Ses derniers mots, on s'en souvient, portent sur le sacrifice que ses amis ne doivent pas oublier de faire à Asclépios, dont le culte ne fut introduit officiellement à Athènes qu'en $420 \mathrm{av}$. J.-C., après la peste qui ravagea la cité entre 430 et 425. Il s'agit donc d'une «nouvelle» divinité de la cité ${ }^{16}$.

Il serait dès lors inapproprié de considérer Socrate comme quelqu'un qui rejette les dieux acceptés par la cité, même s'il paraît porter une attention particulière aux divinités marginales, ou s'il se sert volontiers du terme général theos ou daimon pour définir la divinité, sans donner plus de précisions sur l'identité d'un dieu particulier ${ }^{17}$. Par contre, il est vrai que Socrate revendique, parallèlement, un lien direct et privilégié avec la divinité, ainsi qu'une manière personnalisée de concevoir et d'exprimer sa piété.

Mais sa particularité la plus importante, comme le soulignent E. Derenne et L. Brisson, est que Socrate transforme d'un point de vue éthique les divinités traditionnelles $^{18}$. La manière «socratique» de concevoir la dimension éthique des dieux est conforme à la rationalité (une première approche systématique du sujet s'effectue dans l'Euthyphron). Toute divinité ne peut qu'être parfaitement sage et bonne, juste et bienveillante pour les hommes.

C'est ainsi que la «mission philosophique» de Socrate, présentée comme un don divin aux Athéniens dans l'Apologie, ne peut qu'avoir des conséquences bénéfiques. Si les juges du philosophe ne partageaient pas cet avis, étaitce vraiment parce que le panthéon et le culte officiel de la cité se voyaient

\footnotetext{
13 Platon, Phèdre, 257a-b et 279b-c. Voir aussi A. MOTTE, « La prière du Philosophe chez Platon », in H. LIMET, J. RiES (éds), L'expérience de la prière dans les grandes religions. Actes du Colloque de Louvain-la-Neuve et Liège (22-23 novembre 1978), Louvain-la-Neuve, 1980, p. 173204.

Platon, Banquet, 220d.

15 Id., Phédon, $117 \mathrm{~b}-\mathrm{c}$.

16 Ibid., 118a. Selon J. LABARBE, «Dernières paroles d'anciens Grecs, dernières paroles de Socrate », $B A B$ (1990), p. 189-222, cette manifestation ultime de piété serait liée à l'approche de la fête d'Asclépios, et le coq serait la victime à offrir d'après le règlement officiel. Nous pensons que cette interprétation, aussi intéressante et valide soit-elle, laisse en fait de côté la symbolique supplémentaire que Platon a sans doute voulu y apporter : Socrate pourrait remercier en mourant le dieu de la médecine parce qu'en appliquant un pharmakon (le terme signifie à la fois « poison » et « remède »), celui-ci l'a « guéri » des maux de cette vie, ce qui permettra à l'âme du philosophe de rejoindre librement la divinité pour l'éternité.

${ }^{17}$ Pour l'usage fréquent du terme theos dans l'Apologie, le Criton et le Phédon, voir aussi R. WeIss, Socrates Dissatisfied. An Analysis of Plato's Crito, New York, Oxford Univ. Press, 1998, p. 16 .

8 E. DERENNE, Les procès d'impiété intentés aux philosophes à Athènes au $V^{\text {e }}$ et au IV siècle avant J.-C., Liège/Paris, 1930 (Bibliothèque de la Faculté de Philosophie et Lettres de l'Université de Liège, 95), p. 105 et BrISSON, o.c. (n. 5), p. 54.
} 
offensés par l'attitude religieuse de Socrate ? Certains chercheurs le pensent ${ }^{19}$, mais nous ne trouvons pas que le témoignage de Platon - au moins tel qu'il vient d'être ici rappelé - justifie cette position. La notion d'asebeia pour les juges athéniens porterait surtout sur l'expression externe du respect de la religion officielle. Il en est sans doute très peu parmi eux à avoir compris la portée de la «transformation éthique » révolutionnaire effectuée discrètement par Socrate dans sa propre manière d'envisager les dieux.

Nous penchons plutôt, avec la plupart des commentateurs, pour l'interprétation qui met en avant les motivations politiques des accusations contre le philosophe ${ }^{20}$. Socrate lui-même en paraît conscient dans l'Apologie, quand il souligne les effets subversifs pour l'équilibre social, et néfastes pour lui, que pouvait avoir son elenchos auprès de tous ceux que l'on considérait couramment comme des savants. C'était une attitude critique que les jeunes de la cité s'étaient empressés d'adopter et de répandre, au grand dam de tous ceux qui avaient intérêt à maintenir les doxai traditionnelles mais fausses, garantissant leur crédibilité et leur pouvoir dans Athènes.

Rappelons encore que le décret de Diopeithès contre les athées date de 432 av. J.-C. et que le terminus post quem de la loi athénienne contre l'asebeia se situe en 456 av. J.-C., mais c'est surtout après la guerre du Péloponnèse que des procès d'impiété ont eu lieu ${ }^{21}$, donc quand la cité se trouvait déjà en situation d'instabilité grave sur le plan politique. Les éventuels écarts par rapport aux croyances de la cité devaient à ce moment-là être considérés à la fois comme une trahison envers la cité elle-même et une bybris envers les dieux; une bybris qui risquait d'avoir des conséquences néfastes pour tous, si elle restait impunie.

Il est notable enfin que dans le Criton Socrate, malgré sa condamnation, paraît vouer un respect absolu aux lois de la cité, qu'il présente comme apparentées à la justice divine ${ }^{22}$. Le philosophe souligne son accord avec la législation athénienne, telle qu'elle avait été définie selon les règles de la démocratie. Par contre, dans l'Apologie, il trouve manifestement que ce sont les juges l'ayant condamné qui n'ont pas su appliquer de manière juste cette législation dans son cas précis ${ }^{23}$. Socrate ne s'attaque nulle part au régime, ni au fonctionnement de la cité, auquel il se soumet de son plein gré, mais n'hésite pas à faire usage du droit précieux, accordé par la démocratie ellemême, d'exprimer ouvertement sa critique contre les faiblesses humaines de ses concitoyens.

\footnotetext{
19 Par exemple, voir R. GARLAND, Introducing New Gods. The Politics of Athenian Religion, Ithaca, New York, Cornell Univ. Press, 1992, p. 151.

${ }^{23}$ Id., Apologie, 38c-42a.
} 


\section{Platon face aux citoyens qui s'écartent des croyan- ces officielles dans les cités idéales}

C'est pour tenter des propositions de cités politiquement «saines» et stables, que Platon entreprend de penser l'organisation de la Kallipolis dans la République et, avec une précision encore plus concrète, celle de la cité des Magnètes dans les Lois. Ce dernier dialogue, qui a dû probablement être publié après la mort de l'auteur, se situe, de manière originale, en Crète, où la cité future est censée s'établir. Peut-être la raison essentielle de l'absence de Socrate réside-t-elle dans ce fait ${ }^{24}$.

La législation des Magnètes accorde une grande importance au culte officiel, largement inspiré de la tradition ${ }^{25}$ et se fonde manifestement sur trois principes religieux : a) les dieux sont bons; b) ils prennent soin de nous et c) leur justice est absolument incorruptible ${ }^{26}$. Ces principes, les législateurs tentent très sérieusement de les soutenir avec une argumentation rationnelle élaborée, qu'ils désirent poser comme "préambule» aux lois contre l'impiété, afin de convaincre les citoyens de leur validité.

D'autre part, les peines seront sévères pour les membres de la cité qui ne partagent pas l'une ou l'autre de ces croyances. Platon fait une distinction : pour ceux qui s'en écartent par ignorance, en se moquant du culte officiel, sans être nécessairement des gens injustes, une ré-éducation de cinq ans est prévue initialement dans une prison spéciale, auprès des membres du «collège de veille » ${ }^{27}$, qui gouverne la cité. Ils pourront ensuite soit réintégrer la cité, soit être mis à mort, s'ils persistent à offenser les lois religieuses ${ }^{28}$. Les autres, qui sont complètement corrompus, et parmi lesquels le philosophe inclut d'éventuels tyrans, démagogues, sophistes, généraux sournois et sorciers exploitant l'incrédulité des foules en vue du gain, seront incarcérés à perpétuité dans un lieu désert, coupés de tout contact avec le monde extérieur; après leur mort naturelle leurs corps seront rejetés hors du territoire, avec interdiction à tous de les ensevelir ${ }^{29}$.

Par l'endurcissement du respect de la loi dont Socrate lui-même faisait preuve, Platon se montre effectivement implacable et particulièrement sévère

${ }^{24}$ Chr. Rowe, «Socrates, the laws, and the Laws», in S. SCOLNICOV, L. Brisson (éds), Plato's Laws, From Theory Into Practice. Proceedings of the VI Symposium Platonicum (Jerusalem, 5-10 August 2001), Sankt Augustin, Academia Verlag, 2003 (International Plato Studies, 15), p. 87-97 (p. 92), pense qu'effectivement, le lieu où se déroule le dialogue empêche la présence plausible de Socrate, mais que pour Platon l'Étranger Athénien jouerait le même rôle que son maître même si souvent on a dit voir derrière ce personnage plutôt Platon lui-même.

${ }^{25}$ Voir O. REVERDin, La religion de la cité platonicienne, Paris, De Boccard, 1945.

26 Platon, Lois X, 907b 5-7.

27 Cette traduction à la place de l'habituelle : «conseil nocturne » est une proposition de

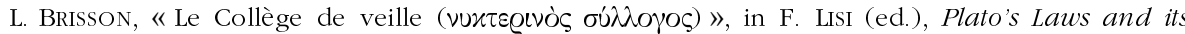
Historical Significance. Selected Papers from the International Congress on Ancient Thought (Salamanca, 1998), Sankt Augustin, 2000, p. 161-177.

28 Platon, Lois X, 908b et 909a.

29 Ibid., 908c-e et 909a-d. 
face aux crimes d'impiété. Qui plus est, la notion de la piété ne se limite pas ici aux manifestations externes du culte, aux us et coutumes, et aux croyances courantes plus ou moins floues, comme c'était le cas dans toutes les cités grecques, mais s'identifie à l'adhésion absolue aux «articles de foi » de la cité. Ces principes, pourtant, sont présentés comme cruciaux pour le maintien du bien-être de l'ensemble de la cité et ils sont définis par les trois législateurs des Lois dialectiquement (même si l'exercice de la dialectique se présente ici dans une forme plutôt élémentaire).

Il est remarquable que ces principes théologiques figurent d'abord dans la République, où c'est Socrate lui-même qui élabore une argumentation rationnelle pour les établir ${ }^{30}$. Ces tupoi peri theologias doivent constituer les règles auxquelles se conformeront les mythes sur les dieux que les poètes feront circuler dans la Kallipolis, car l'éducation éthique des gardiens de la cité (et, en l'occurrence, de tous les citoyens) ne peut pas être compromise par des croyances religieuses irrationnelles et des modèles de comportement immoraux.

Si, dans la République, Socrate en tant que législateur potentiel essaie d'éviter ce qu'il estime néfaste dans les croyances de la cité, dans les Lois, les trois vieillards prennent des mesures pour affronter ce même mal, au cas où il se présenterait. Mais nous ne pensons pas qu'il y ait des différences concernant les principes théologiques «officiels» entre les cités idéales des deux dialogues. Il est évident que Platon désire garder l'ensemble des citoyens attachés à tout prix à ces principes, surtout parce que, lui, il estime que la survie et la bonne vie de la cité en dépendent ${ }^{31}$. Ces croyances rationnellement définies sont présentées en même temps comme inspirées par la divinité; dès lors, elles sont censées refléter la justice divine elle-même.

Platon se trouve ainsi finalement d'accord avec les Athéniens de son époque sur le principe selon lequel l'ensemble des citoyens doit adopter des attitudes religieuses communes, en vue d'assurer la stabilité politique. Il va même plus loin qu'eux, en attachant une importance capitale à la conviction interne, dont les manifestations cultuelles ne sont que l'expression superficielle. Les croyances religieuses concernant la nature des divinités et les rapports de celles-ci avec les hommes n'étaient jamais aussi clairement établies dans la religion des cités grecques réelles. C'est la définition rationnelle des principes théologiques et leur accord avec le bien qui différencie essentiellement la position du philosophe et ne laisse plus aucun espace aux croyances individualisées.

\footnotetext{
30 Platon, Répubique II, 378e sq.

31 Voir aussi T.J. SAUNDERS, «Plato on the treatment of heretics », in L. FOXHALL, A.D.E. LEWIS (éds), Greek Law in its Political Setting: Justifications, not Justice, Oxford, 1996, p. 301-323; M. SCHOFIELD, «Religion and philosophy in the Laws », in SCOLNICOV - BRISSON (eds), o.c. (n. 24), p. $1-13$ (p. 13)
} 


\section{Conclusions}

Après ces clarifications, que peut-on conclure relativement aux attitudes respectives de Socrate et de Platon lui-même sur ce sujet?

Socrate, tel qu'il se présente dans la République, est-il en accord avec le protagoniste de l'Apologie, du Criton ou du Phédon, mort pour cause d'impiété ? Approuverait-il les mesures contre les impies proposés par l'Athénien des Lois ? Platon a-t-il complètement oublié la condamnation de son maître à la fin de sa propre vie?

Même si on peut avoir de bonnes raisons de nier la réelle élaboration d'une cité idéale par Socrate lui-même, nous ne pensons pas que les principes religieux que le philosophe suivait, tels qu'on peut les voir dans les dialogues qui décrivent sa défense au tribunal et sa fin, sont en désaccord avec ceux qui figurent, sous une forme plus claire et plus élaborée, dans la République et les Lois.

La manière dont Platon en tant que législateur traite les membres de la cité qui n'adhèrent pas à ses conceptions religieuses est sans aucun doute plus dure que celle qu'appliquait Socrate, comme on le voit dans l'Euthyphron. Platon ne se donne plus la peine de faire patiemment le cheminement dialectique personnalisé avec chacun des membres des cités idéales. Il présente seulement à la population l'ensemble de ce parcours déjà effectué par des personnes sages et vertueuses. À l'instar de Socrate, les législateurs de la cité des Magnètes jouissent apparemment d'une certaine communication privilégiée avec la divinité.

Persuadé que les croyances définies ainsi par le logos à la fois rationnel et inspiré représentent le bien véritable, Platon identifie la liberté personnelle (liberté par rapport aux doxai fausses et aux faiblesses humaines) à l'acceptation volontaire et consciente de celles- $\mathrm{ci}^{32}$. Socrate en espérait autant, quand il exerçait son elenchos auprès des Athéniens en vue de les amener à découvrir rationnellement la vérité par eux-mêmes. Pour lui, «personne n'était méchant de son plein gré». Son élève se permet de se servir du pouvoir législatif pour imposer ces positions en cas de désaccord, qu'il envisage également comme dû à un dysfonctionnement psychique chez la personne concernée, résolument nuisible pour la société.

Les points de vue adoptés par Socrate et par Platon divergent par rapport à la manière d'avancer leurs avis pour les faire accepter par les citoyens. Ceux-ci sont, d'ailleurs, des membres de cités aux régimes différents, auxquels l'un adresse comme à des égaux et l'autre en tant que fondateur de leur législation. Or, la définition dialectique et rationnelle des croyances sur les dieux, leur accord nécessaire avec le bien, ainsi que leur importance éducative, éthique et politique, sont un héritage socratique qui reste présent dans l'ensemble de l'œuvre platonicienne.

32 Pour la problématique autour de l'autorité religieuse et de la liberté dans la cité des Magnètes, ainsi que pour une tentative d'interprétation, voir A. LEFKA, "Souveraineté divine et liberté humaine dans les Lois », in SCOLNICOV - BRISSON (eds), o.c. (n. 24), p. 155-164. 
Nous irons encore plus loin, en soutenant que dans ces cités idéales, Platon accorde au philosophe, dont l'incarnation archétypale est pour lui Socrate, le pouvoir que celui-ci n'avait jamais eu à Athènes. Dans cet esprit, Socrate ne serait pas un étranger dans la cité des Magnètes, comme l'ont souvent dit divers chercheurs ${ }^{33}$, mais devrait siéger au "Collège de veille ", faisant même peut-être partie de ces membres de la cité excellents en sagesse et en vertu, les euthynoi, qui étaient consacrés à Apollon et à Hélios et qui vivaient aux frais de la cité dans le temple commun de ces dieux, au coeur de l'Agora $^{34}$. Socrate ne disait-il pas dans l'Apologie qu'être nourri par la cité dans le Prytanée aurait dû être sa récompense pour les services offerts à Athènes par la volonté d'Apollon ${ }^{35}$ ?

Loin d'oublier son maître, Platon à la fin de sa vie crée une cité qui lui accorderait tout l'honneur dont il était digne, en suivant ses propres principes religieux et philosophiques. Ceux qui se trouveraient au banc des accusés seraient dès lors, par un renversement des rôles, des hommes dont les croyances n'étaient pas en accord avec le logos, comme, par exemple, Anytos et Mélétos.

Nous trouvons même dans le texte des Lois, au sein de l'argumentation sur les principes religieux officiels, une référence furtive, mais particulièrement indicative, qui montre bien, à notre avis, que le souvenir de la condamnation de Socrate reste toujours aussi vivant pour son élève, malgré le temps écoulé depuis $^{36}$ :

(L'Athénien :) Alors, quoi ? Que disons-nous ? Que devons-nous faire? Nous défendrons-nous comme des gens qu'on poursuivrait devant un tribunal d'impies, ceux-ci disant aux persécutés de notre législation que nous faisons des choses terribles en y décrétant l'existence des dieux?

En conclusion, nous sommes d'avis que, dans ces cités idéales, Platon rend publiques les croyances personnelles de Socrate (lequel n'avait jamais accepté ouvertement un désaccord de sa part avec la législation d'Athènes en matière de religion). Ensuite, il se montre radicalement intolérant face aux attitudes religieuses courantes de ses propres concitoyens qui ne résisteraient pas à l'elenchos rationnel. En cela, il aurait même recours - en dernier lieu, cependant - à la force, s'il avait accès à un pouvoir politique que Socrate n'a jamais cherché à acquérir, se contentant de la persuasion par le dialogue personnel et égalitaire. Il est fort possible que la véhémence de Platon contre les croyances qu'il marginalise dans ses cités idéales, parce qu'il les estime superficielles, fausses et dangereuses, soit due en partie à sa révolte de

33 Cf., par exemple, Strauss, o.c. (n. 4) et Rowe, o.c. (n. 24).

Platon, Lois XII, 945b-947e; pour le parallèle entre Socrate et les euthynoi, voir aussi A. LEFKA, «Au service des dieux et des hommes. Etre prêtre dans la cité de Platon », Kernos 9 (1996), p. 129-143 (p. 142-143).

Platon, Apologie, 36d-37a.

36 Ibid., 886e 7-887a 2 (trad. A. Diès, CUF). 
jeunesse, toujours vivace, devant la condamnation injuste de son maître par les représentants de ce genre de croyances.

Malgré les apparences, nous pensons que Platon veut sincèrement s'allier à Socrate, à sa façon, en vue de voir se répandre dans la cité une religiosité solidement définie par le logos, sur lequel se fonderait, de la meilleure manière possible, l'éducation éthique de chacun des citoyens et, par conséquent, la vraie bonne vie de la citée . $^{37}$.

Ce qui distingue essentiellement Platon de Socrate, tel que lui-même le présente surtout dans ses dialogues dits «de jeunesse », ainsi que des conceptions courantes athéniennes, est qu'il exclut fermement toute possibilité de choix ou d'interprétations personnelles en matière de religion. La démocratie athénienne, acceptée par Socrate comme le meilleur régime sous lequel il pouvait vivre, présentait, malgré tout, une plus grande souplesse, ce que la religion traditionnelle permettait aisément. Mais Platon pensait que le régime idéal serait une «timocratie», où le logos vrai et la vertu que celui-ci engendre gouverneraient sans conteste. On pourrait dire qu'il s'agit d'une poussée à l'extrême des conceptions socratiques, auxquelles est accordée la prééminence dans l'organisation de la cité.

L'appel personnalisé du maître garde-t-il encore toute sa valeur quand il devient une institution incontournable grâce au zèle du disciple? Nous ne saurions l'affirmer, car sur cette question nous avons, quant à nous, quelques sérieuses réserves...

Aikaterini LEFKA

Université de Luxembourg / Université de Liège

Boulevard d'Avroy, 77/111

B - 4000 LIÈGE

\footnotetext{
37 Cf. aussi MOTTE, l.c. (n. 1), p. 47 : « La défense de Socrate y était toute entière bâtie sur l'idée d'un lien privilégié et purement personnel unissant la divinité et le philosophe dans la défense du bien moral. Mais c'était au prix d'un divorce avec la cité. Désormais, la transcendance reconnue aux Lois de la patrie (dans le Criton) suggère qu'un tel lien, s'il libère et conforte puissamment la conscience du sage, n'ignore pas pour autant la médiation du collectif. Mais comment réconcilier la psychè et la polis? Telle sera, sa vie durant, la quête inachevée de Platon. » Nous pensons que finalement Platon a pu trouver un moyen d'achever sa quête, en imposant la vision du sage à la collectivité dans ses cités idéales.
} 